M.Sc. Andrea Sconosciuto, https:/ / orcid.org/0000-0002-9529-5141

Pontificia Universitas Lateranensis

\title{
Amor coniugalis e matrimonio canonico
}

\section{Miłość małżeńska i małżeństwo kanoniczne Conjugal love and canonical marriage}

\author{
https://doi.org/10.34766/fetr.v45i1.704
}

\begin{abstract}
Nowość wprowadzona przez Sobór Watykański II odnośnie do istoty małżeństwa przyniosła wielkie zmiany w redakcji Kodeksu Prawa Kanonicznego z 1983 roku. W poprzednim Kodeksie z 1917 roku, cele i istota małżeństwa nie miały ściśle określonych granic, wręcz przeciwnie, dość często były one mylone pomiędzy sobą. Małżeństwo było badane tylko w perspektywie kontraktu i celu. Wizja personalistyczna, wprowadzona przez Sobór Watykański II, wprowadziła nową koncepcję istoty małżeństwa. U podstaw refleksji nad instytucją małżeństwa została postawiona miłość małżeńska. Intymna komunia miłości staje się komórką kształtującą życie małżeńskie, a droga małżonków w życiu małżeńskim staje się duchowym i czynnym wzrostem w miłości. W całej pełni, zgodnie z nauczaniem soborowym, posynodalna Adhortacja papieża Franciszka Amoris laetitia nadaje elementum amoris konotację historyczną, teraźniejszość i przyszłość w rozwoju rodziny i życia małżeńskiego. Małżeństwo staje się wypełnieniem planu Boga wobec człowieka.
\end{abstract}

Słowa kluczowe: małżeństwo, miłość małżeńska, istota małżeństwa, prawo kanoniczne

Abstract: The innovation introduced by Vatican Council II about the research on marriage led great changes in the 1983 edition of the Canon Law of the Catholic Church. The former 1917 Canon Law the purpose and the essence of marriage lacked well-defined boundaries; indeed, the two concepts were often confused. Marriage was only viewed as a contract with a purpose. The personalistic vision developed by the Vatican Council introduced a new conception of the essence of marriage. Marital love became the centre of married life while the path of the spouses in married life is seen as a spiritual and factual growth in love. In full compliance with the conciliar teaching, the post synodal Apostolic Exhortation Amoris Laetitia of Pope Francis confers to "elementum amoris" a historical connotation, a present and a future in the growth of family and matrimonial life. Marriage becomes fulfilment of God's plan for man.

Keywords: marriage, marital love, essence of marriage, canon law

1. «Itaque iam non sunt duo sed una caro. Quod ergo Deus coniunxit, homo non separet» (Vangelo di Matteo 19, 6)

Se dovessimo dare una definizione di matrimonio, forse, l'unico riferimento a cui tendere sarebbe proprio il passo del Nuovo Testamento appena citato. Una sola carne indica proprio il concetto matrimoniale della dedizione tra i coniugi nella perfetta comunità di amore, costituita davanti a Dio mediante la presenza di un ministro ordinato che riceve il consenso.

Un antico brocardo latino citava "omnis definitio in iure periculosa est", infatti anche il legislatore canonico ha lasciato le definizioni giuridiche alla dottrina evitando di inserirle nel codice. Una scelta di sintesi, forse, ma puramente codiciale ed efficace. La legge 
matrimoniale non deve definire, ma deve regolamentare, disciplinare e normare gli atti che vengono compiuti; a maggior ragione atti tali da comportare una modificazione nello status personae.

Il Titolo VII del libro IV del Codice di diritto canonico, dedicato al matrimonio, ha forte rilevanza, non solo giuridica, ma anche sociale. Il matrimonio, infatti, non è solamente una realtà religiosa, ma compenetra l'essere umano in ogni suo aspetto, anche civile e sociale. Per i cristiani, la natura sacramentale del matrimonio acquista una rilevanza precipua, per il particolare status che conferisce, ma nel pensiero e nella conformazione giuridica di questo istituto confluiscono varie materie, dalla filosofia, alla scienza, alla psicologia, ciascuno con il proprio contributo fondamentale) (Abate, 1985, 1986; Aznar, 1985; Bertolino, 1996, Bianchi, 1990; Bonnet, 1996; Hervada, 2000; Moneta, 1992; Pinto, 2001).

Come si è giunti alla definizione del matrimonio canonico così come lo intendiamo oggi? Quali insegnamenti la Chiesa ci ha dato affinché tale istituto, importantissimo nella vita laicale possa essere valido ed efficace e possa dar continuo vigore alla Chiesa? Come il Concilio Vaticano II ha completamente stravolto il focus dell'indagine sul matrimonio? Quali novità introduce l'Amoris Letitia?

Una bella sfida, ma allo stesso tempo una pagina bellissima della storia della Chiesa e della sua riflessione teologica. Non dimentichiamo che il codice di diritto canonico, promulgato da S. Giovanni Paolo II con la Costituzione Apostolica Sacrae Disciplinae Legis del 25 gennaio 1983, costituisce l'ultimo documento del Concilio Vaticano II ${ }^{1}$.

Se per tutti gli altri sacramenti il legislatore ha voluto dare una definizione giuridica degli elementi essenziali, circa il matrimonio non ci sono definizioni vere e proprie (Sabbarese, 2019, p. 41).

Il can. 1055 dimostra quanto la materia matrimoniale sia allo stesso tempo giuridica, sacramentale e fondata su un sottile equilibrio che solo nella Chiesa poteva essere fondante: l'amore tra i due coniugi.

«Matrimoniale foedus, quo vir et mulier inter se totius vitae consortium constituunt, indole sua naturali ad bonum coniugum atque ad prolis generationem et eductionem ordinatum, a Christo Domino ad sacramenti dignitatem inter baptizatos evectum est» ${ }^{2}$ (\$1). Ogni termine è soppesato, ogni parola ben ponderata con logica e alla luce del Magistero della Chiesa.

Come già si evince dai primi due termini, il matrimonio viene visto come un "foedus", un patto, ma non un semplice patto tra persone: implica una scelta di vita. Il legislatore, infatti, non ha utilizzato il termine "pactum", ma "foedus": con alcune reminiscenze stoiche si può ben ricordare l'Europa medievale quando il territorio del Sacro Romano Impero era diviso in Feudi. Il termine feudo significa proprio patto, ma un patto che

1 http://www.vatican.va/content/john-paul-ii/en/apost_constitutions/documents/hf_jpii_apc_25011983_sacrae-disciplinae-leges.html

${ }^{2}$ http://www.documenta-catholica.eu/d_1983-01-25-\%20Codex\%20Iuris\%20Canonicis\%20-

\%20LT.pdf 
legava a vita il signore con il suo vassallo: se questo tradiva il patto si macchiava del reato di fellonia.

Il patto che i due coniugi vanno a costituire è un patto vitalizio, che solo per conclusione naturale di esso si può sciogliere.

Questo patto tra uomo e donna ha la funzione di "consortium", una piccola societas volta al mutuo aiuto negli obblighi e doveri e ordinato al bene dei coniugi, alla generazione e alla educazione della prole.

Come Cristo ha amato e ama la sua sposa, la Chiesa, così l'amore di Dio verso l'uomo si alimenta nell'amore che i coniugi si promettono per tutta la vita e che riversano nei loro figli. Un continuo scambio di amore che alimenta i fedeli e che alimenta tutta la Chiesa. In questo "admirabile commercium" si trova proprio il fondamento di quella Oikonomia della Salvezza.

San Giovanni Paolo II nella Esortazione Apostolica Familiaris Consortio ha affermato: «Nella sua realtà più profonda, l'amore è essenzialmente dono e l'amore coniugale, mentre conduce gli sposi alla reciproca conoscenza che li fa una carna sola, non si esaurisce all'interno della coppia, poiché li rende capaci della massima donazione possibile, per la quale diventano cooperatori di Dio per il dono della vita ad una nuova persona umana. Così i coniugi mentre si donano tra loro, donano al di là di se stessi la realtà del figlio, riflesso vivente del lor amore, segno permanente della unità coniugale e sintesi viva ed indissociabile del loro essere padre e madre» (Giovanni Paolo II, 1981, n. 14).

Perché il matrimonio sia costituito, un elemento essenziale è il consenso manifestato pienamente e liberamente dai coniugi, che abbraccia gli elementi e le proprietà essenziali del matrimonio, nonché gli obblighi derivanti da esso. Il canone 1057 recita che «matrimonium facit consensum», una traditio già individuata dai romani che avevano individuato nel consenso il fondamento del matrimonio.

Poiché il consenso dato e scambiato è fondativo del matrimonio, questo deve essere pieno e consapevole su ciò che si va a scegliere.

Prestare il consenso è l'atto umano che implica proprio uno status di vita. Rimane proprio in questo contesto sempre più attuale la differenza tomistica dell'atto dell'uomo e dell'atto umano: il primo riguarda atti che possono essere comuni anche con gli animali, provengono da noi senza però essere ontologicamente umani; il secondo riguarda gli atti che rivelano l'uomo in quanto uomo, soggetto dotato di elemento razionale (Leonard, 2001, p. 26).

Nell'atto positivo dell'accettazione degli elementi, delle proprietà essenziali e degli obblighi del matrimonio, i coniugi compiono l'atto umano per eccellenza, quello che implica la nascita di una nuova comunità. 
2. Nel codice pio-benedettino del 1917 la concezione dell'essenza del matrimonio era di ordine strettamente giuridico - contrattualistico, infatti, essa consisteva nella deditio iuris in corpus perpetui et exclusivi. Dando il loro consenso, i nubenti manifestavano la volontà di donarsi reciprocamente il diritto di compiere, l'uno sul corpo dell'altro, atti idonei alla procreazione della prole, in perpetuo, cioè fino a che il vincolo rimanesse in vita, ed in modo esclusivo, vale a dire escludendo terzi dal compimento di tali atti (Piana, 1994). La concezione dell'essenza del matrimonio era prettamente finalistica.

Nella dottrina canonistica del vigente codice del '17 mancava una vera e proprio dottrina specifica dell'essenza del matrimonio, dato che l'indagine si dirigeva in maniera esclusiva sui fini e sulle proprietà essenziali del matrimonio, dando rilievo assoluto al tema della procreazione come fine primario dell'unione. Si creava una sorta di confusione tra l'essenza e i fini del matrimonio.

Il risultato che ne viene fuori è la produzione di un modello ove la priorità viene assegnata agli aspetti formali, o meglio, giuridico-istituzionali, tralasciando la visione antropologica del matrimonio. Ciò porta ad una disattenzione verso l'essenza del matrimonio e della coppia stessa nel suo farsi una caro, negando qualunque tipo di riflessione sulla realtà dell'amore interpersonale.

La svolta interpretativa sull'essenza del matrimonio si è avuta con la celebrazione del Concilio Vaticano II, che ha dato al matrimonio una visione personalistica.

Il focus dell'indagine antropologica, giuridica e teologica sull'essenza del matrimonio è la coppia, colta in tutte le espressioni relazionali e vitali.

La comunione di vita dei coniugi non viene vista più come un fine, ma come l'essenza del matrimonio stesso. L'analisi si sposta dalla prospettiva finalistica del vecchio codice alla visione antropologica e personalista: dal sinallagma delle prestazioni reciproche si passa al piano dell'elementum amoris che unisce due persone.

La rivoluzione della riflessione ecclesiale sul matrimonio data dal Concilio sta proprio nel concetto di amor coniugalis. Esso non è inteso in senso soggettivistico, ma personalistico: dall'amore la coppia si nutre, come scambio reciproco tra due persone, intese nella loro intima comunità di vita e non soltanto come scambio di atti idonei alla procreazione.

Accanto ai tria bona agostiniani (bonum sacramenti, bonum prolis e bonum fidei) per la prima volta, dopo le riflessioni conciliari, si inserisce il bonum coniugum, il bene dei coniugi. Il concilio, con la sua visione innovativa e personalista, ha voluto dare al sacramento matrimoniale non una visione puramente finalistica, ma ha voluto che tale sacramento fosse fondato sull'intima comunità di vita e di amore che si instaura tra i coniugi (Gaudium et spes, n. 48) (Wegan, 1988).

In ciò risiede l'essenza del matrimonio.

Se per essenza deve essere inteso ciò che identifica una cosa in se stessa, comprendendo tutti e solo gli elementi necessari ad individuarla nella sua entità e nel suo 
insieme, l'elementum amoris è essenza del matrimonio, perché senza l'amore la coppia non esisterebbe. Beninteso, Dio è amore, come affermato nella lettera di Giovanni, senza l'amore di Dio l'uomo sarebbe nulla. La proiezione di quell'agape divino sull'uomo, fa sì che anche l'uomo possa vivere dell'amore che lui prova nei confronti del suo coniuge.

L'atto di amore della donazione di se stessi è l'atto di amore più grande: dalla donazione di Cristo sulla croce, da quelle braccia allargate, il mondo è pervaso di amore. Il matrimonio trova fondamento nell'Amore Pasquale, diviene quasi segno della Chiesa, nella quale un tale amore viene costantemente riversato ed è proiettato verso la pienezza della salvezza, dove troverà la definitiva consumazione (Pellegrino, 1998).

Oggetto specifico del consenso dei coniugi non sarà più il ius in corpus, ma «sese mutuo tradunt et accipiunt ad consituendum matrimonium», come citato dal can. 1057: i coniugi si danno e si accettano reciprocamente in una comunità intima di amore, così come Dio è intimamente unito nell'amore verso l'uomo (Bonnet, 1985, p. 35).

3. Come Cristo ha amato la sua unica Chiesa, così anche il matrimonio ha come proprietà essenziale l'unità. Questa consiste nell'unione di un solo uomo con una sola donna. Il matrimonio ha anche come proprietà l'indissolubilità, dura cioè per tutta la vita e non può sciogliersi se non per la morte di uno dei coniugi.

Lo stesso canone 1141 afferma: «Matrimonium ratum et consummatum nulla humana potestatem nullaque causa, praeterquam morte, dissolvi potest ${ }^{3}$.

Il consenso dato dai coniugi nell' atto del matrimonio diventa irrevocabile e il vincolo non può essere sottoposto a null'altra decisione o potere dei coniugi stessi.

San Giovanni Paolo II, sempre in Familiaris Consortio afferma: «La comunione coniugale si caratterizza non solo per la sua unità, ma anche per la sua indissolubilità. È dovere della Chiesa riaffermare con forza la dottrina dell'indissolubilità del matrimonio: a quanti ai nostri giorni ritengono difficile o addirittura impossibile legarsi ad una persona per tutta la vita e a quanti sono travolti da una cultura che rifiuta l'indissolubilità e che deride apertamente l'impegno degli sposi alla fedeltà, è necessario ribadire il lieto annuncio della definitività di quell'amore coniugale, che ha in Gesù Cristo il suo fondamento e la sua forza. Radicata nella personale e totale donazione dei coniugi e richiesta dal bene dei figli, l'indissolubilità trova la sua verità ultima nel disegno che Dio ha manifestato nella sua Rivelazione» (San Giovanni Paolo II, Familiaris Consortio, n. 20).

È da Cristo che si riceve la grazia nel nutrimento quotidiano all'amore anche tra coniugi. Non a caso nella formula del consenso matrimoniale leggiamo: «Io N., accolgo te N., come mia sposa/o. Con la Grazia di Cristo prometto di esserti fedele sempre...» ${ }^{4}$.

3http://www.documenta-catholica.eu/d_1983-01-25-\%20Codex\%20Iuris\%20Canonicis\%20-

\%20LT.pdf

${ }^{4}$ http:// famiglia.chiesamodenanonantola.it/2004/10/20/nuovo-rito-del-matrimonio/ 
Il progetto di Dio sull'uomo si basa sull'amore e tutta la storia della salvezza, che passa anche attraverso l'amore tra uomo e donna, non è altro che un atto donativo assoluto.

È in questo solco che si instaura la visione che il Romano Pontefice Francesco ha voluto dare, al contempo, alla pastorale matrimoniale e familiare.

Nell'Esortazione Apostolica Amoris Laetitia l'amore prende forma nella sua storicità. Dio non rinuncia all'alleanza con l'uomo, ma nutre del suo amore la storia dell'uomo facendosi dono anche nella famiglia (Francesco, 2016).

«Il matrimonio è un segno storico per coloro che camminano sulla terra» afferma il Sommo Pontefice al n. 161: l'amore è un percorso che l'uomo compie e allo stesso modo il matrimonio è un «cammino permanente di crescita», un cammino che istruisce e che richiama ad una «costante maturazione» (Francesco, 2016).

Nella riflessione condotta dal Romano Pontefice Francesco in Amoris Laetitia, l'elementum amoris, espresso dai padri conciliari e predicato dai Pontefici predecessori, trova la sua massima collocazione nella storia dell'uomo. L'amore viene visto come un percorso, un cammino tipico dell'uomo che comporta necessariamente una crescita nelle relazioni e nell'amore stesso.

Così come il fidanzamento è un percorso nell'amore in preparazione alla celebrazione del sacramento del matrimonio, così il matrimonio stesso non deve essere visto come un punto di arrivo, statico e informe nella storia, ma un perenne cammino di crescita e di miglioramento nelle relazioni familiari.

Occorre ripensare il cammino di preparazione al matrimonio, inteso non soltanto come una formazione prossima alla celebrazione del sacramento (corso prematrimoniale): ogni fedele laico dovrebbe, sulla base delle proprie inclinazioni, scegliere la propria vocazione e il proprio status all'interno della societas Chiesa. Prepararsi al matrimonio richiede la capacità di essere consapevoli nell'accettare l'altro per tutta la vita e solo l'amore verso l'altro può essere aiuto in questa crescita continua.

Sovente ci si interessa al matrimonio solo nella fase patologica di questo: le difficoltà matrimoniali, la difficile convivenza fino ad arrivare ai processi di nullità matrimoniali. Occorre ripensare, sulla base del magistero, ad una corretta pastorale matrimoniale che ponga il consortium totius vitae al centro della riflessione.

Come afferma il Pontefice, nel matrimonio: «L'unione è reale ed irrevocabile...ma nell'unirsi gli sposi diventano protagonisti, padroni della propria storia e creatori di un progetto che occorre portare avanti insieme" (Francesco, 2016, n. 218). La dimensione matrimoniale, basata sull'amore interpersonale tra i coniugi, assume un connotato dinamico, così come già delineato in Familiaris Consortio: «...Si sviluppa così un processo dinamico, che avanza gradualmente con la progressiva integrazione dei doni di Dio e delle esigenze del suo amore definitivo ed assoluto nell'intera vita personale e sociale dell'uomo» (Giovanni Paolo II, 1981, n. 9). 
Il percorso della vita matrimoniale si nutre e si compenetra nell'amore, così come tutta la storia della salvezza è riflessione di quell'amore incondizionato di Dio Padre verso l'uomo.

Nella situazione attuale appare difficile fare questo tipo di considerazioni, nella mentalità odierna si rischierebbe di pensare anacronisticamente, ma la sfida della Chiesa è proprio quella di ribadire la ricchezza del suo depositum fidei. Anche dal punto di vista giuridico sembrerebbe quasi imbarazzante far poggiare un istituto giuridico, come il matrimonio, su di un elemento effimero come un sentimento, ma la ricchezza del magistero e della Parola rafforzano il cammino di chi crede, accettando anche le sfide di un mondo che cambia sempre più repentinamente.

\section{Bibliografia:}

Abate, A. (1985). Il matrimonio nella nuova legislazione canonica, Roma: Urbaniana University Press.

Abate, A. (1986). Il consenso matrimoniale nel nuovo Codice di Diritto Canonico, Apollinaris, $59,445-491$.

Aznar-Gil, F.R. (1985). El nuevo derecho matrimonial canónico, Salamanca: Publicaciones de la Universidad Pontificia de Salamanca.

Bertolino, R. (1996). Gli elementi costitutivi del bonum coniugum: stato della questione, (in:) Il bonum coniugum nel matrimonio canonico, 7-32, Città del Vaticano.

Bianchi, E. (1990). E' più facile con nuovo codice di diritto canonico dimostrare la nullità di un matrimonio?, QDE, 3, 394-410.

Bonnet, P.A. (1985). Introduzione al consenso matrimoniale canonico, Milano: Univ. Milano-Fac.

Bonnet, P.A. (1996). Il bonum coniugum e l'essenza del matrimonio, (w:) Il bonum coniugum nel matrimonio canonico, 89-135, Città del Vaticano.

Castaňo, J.F. (1990). El canon 1057, centro de la legislación matrimonial de la Iglesia, REDC, 47, 563-575.

Castaño, J.F. (1992). Il Sacramento del matrimonio, Roma.

Chiappetta, L. (2011). Il codice di diritto canonico, Commento giuridico-pastorale, Bologna: EDIZIONI DEHONIANE BOLOGNA.

Concilium Oecumenicum Vaticanum II (1965). Constitutio Pastoralis de ecclesia in mundo huius temporis Gaudium et Spes.

D'Auria A. (2007). Il consenso matrimoniale. Dottrina e giurisprudenza canonica, Roma: Aracne.

Francesco, (2016). Esortazione Apostolica post sinodale Amoris Laetitia, Città del Vaticano.

García, Failde J.J. (1986). Observationes novae circa matrimonium canonicum simulatum et coactum, Periodica, 75, 171-220.

García, M.J. (1996). Le norme generali del Codex Iuris Canonici, Roma: EDIURCLA. 
Giovanni Paolo II, (1981), Esortazione Apostolica Familaris Consortio.

http://www.vatican.va/content/john-paul-

ii/it/apost_exhortations/documents/hf_jp-ii_exh_19811122_familiaris-

consortio.html

Hervada, J. (2000). Studi sull'essenza del matrimonio, Milano: Giuffrè.

Lèonard, A. (2001). Il fondamento della morale, Cinisello Balsamo (MI).

Moneta, P. (1992). Il Matrimonio, (w:) Il Diritto nel Mistero della Chiesa, t. 3, 165-302, Roma.

Pellegrino, P. (1998). Il consenso matrimoniale nel codice di diritto canonico latino, Torino.

Piana, G. (1995). La coppia nel Rito-Sacramento cristiano, (w:) La celebrazione del matrimonio (cammino antropologico ed esperienza di fede), Padova.

Pinto, P.V. (2001). Commento al Codice di Diritto Canonico, Città del Vaticano.

Sabbarese, L. (2019). Il matrimonio canonico nell'rodine della natura e della grazia, Città del Vaticano.

Vitalia, E., Berlingò, S. (1994). Il matrimonio canonico, Milano.

Wegan, M. (1988). L'esclusione del «bonum fidei» nella più recente giurisprudenza della Rota Romana, Apollinaris, 61, 89-107. 